\title{
Synthesis and Investigations of Antimicrobial, Antioxidant Activities of Novel Di-[2-(3-alkyl/ aryl-4,5-dihydro-1H-1,2,4-triazol-5-one-4-yl)- azomethinephenyl] Isophtalates and Mannich Base Derivatives
}

\author{
Haydar Yüksek¹, Gül Özdemir¹, Sevda Manap', Yonca Yılmaz', Gül Kotan²*, Özlem \\ Gürsoy-Kol'1, Muzaffer Alkan ${ }^{3}$ \\ 1 Kafkas University, Department of Chemistry, Chemistry, Kars, Turkey \\ 2 Kafkas University, Kars Vocational School, Chemistry, Kars, Turkey \\ 3 Kafkas University, Faculty of Education, Mathematics and Science Education, Kars, Turkey
}

\begin{abstract}
In this study, the synthesis of di-[2-(3-alkyl/aryl-4,5-dihydro-1H-1,2,4-triazol5-one-4-yl)-azomethinephenyl] isophtalates (2a-g) from the reactions of 3 -alkyl/ aryl-4-amino-4,5-dihydro- $1 H$-1,2,4-triazol-5-ones (1a-g) with di-(2-formylphenyl) isophtalate is described. Then, the compounds 2 were treated with morpholine in the presence of formaldehyde to synthesize di-\{2-[1-(morpholine-4-yl-methyl)-3alkyl(aryl)-4,5-dihydro- $1 H$-1,2,4-triazol-5-one-4-yl]-azomethinephenyl $\}$ isophtalates (3a-g). The newly synthesized compounds were characterized using IR, ${ }^{1} \mathrm{H}$ NMR and ${ }^{13} \mathrm{C}-\mathrm{NMR}$ spectral data. In addition, the compounds synthesized were screened for their antimicrobial activities. Furthermore, the antioxidant properties of the newly synthesized compounds were analysed for their in-vitro potential antioxidant activities in three different methods (reducing power, free radical scavenging and metal chelating activity). These antioxidant activities were compared to those from standard antioxidants, such as BHA, BHT, EDTA and $\alpha$-tocopherol.
\end{abstract}

Keywords: Schiff base, Mannich base, Antimicrobial activity, Antioxidant activity.

\footnotetext{
*Corresponding Author: Gül Kotan, e-mail: gulkemer@hotmail.com Haydar Yüksek ORCID: 0000-0003-1289-1800

Gül Özdemir ORCID: 0000-0001-7823-0920

Sevda Manap ORCID: 0000-0002-5025-9622

Yonca YIImaz ORCID: 0000-0002-6536-9269

Gül Kotan ORCID:0000-0002-4507-9029

Özlem Gürsoy-Kol ORCID: 0000-0003-2637-9023

Muzaffer Alkan ORCID: 0000-0002-8820-4555

(Received 27 August 2019, accepted 20 October 2019)
} 


\section{INTRODUCTION}

A large number of heterocyclic compounds containing the 1,2,4-triazole ring, are associated with diverse biological properties such as antioxidant, anticonvulsant, anti inflammatory, antimicrobial and anti-viral activity. Mannich bases have applications the field medicinal chemistry, the product synthetic polymers, the petroleum industry, as products used in water treatment, cosmetics, the dyes industry, etc ${ }^{1}$. In addition, Mannich bases have biological activity such as anticancer ${ }^{2,3}$, antibacterial ${ }^{4-6}$, antimycobacterial ${ }^{7-9}$, anti inflammatory ${ }^{10-12}$, analgesic ${ }^{13,14}$, antifungal ${ }^{15,16}$, antitumor ${ }^{17,18}$ namely the 1 -aryl2-dimethylaminomethyl-2-propen-1-one hydrochlorides 1a-e and 1-aryl-3-dimethylamino-2-hydroxymethyl-1-propanone hydrochlorides 2a-e. A number of these compounds possess marked cytotoxic potencies (IC(50, antiviral ${ }^{19-21}$, antidepressant ${ }^{22,23}$, antiulcer ${ }^{24}$, anticonvulsant ${ }^{25}$, antimalarial ${ }^{26,27}$ and antioxidant activities ${ }^{28}$.

Antioxidants were defended organisms and cells from damage induced by oxidative stress. Thus, significant research has been ruled to investigate this feature. Scientists have dealt with the new compounds in recent years. Natural sources provide the effective components forestall or decrease the influence of oxidative stress on cells have been used ${ }^{29}$. Exogenous chemicals and endogenous metabolic steps in human body or in food system might produce highly reactive free radicals, particularly oxygen provided radicals, which are capable of oxidizing biomolecules, resulting in cell death and issue damage. Oxidative damages play a considerable pathological role in human diseases. It has been an important pathological effect of oxidative damage in human disease. For example, cancer, emphysema, cirrhosis, atherosclerosis and arthritis have all been correlated with oxidative injury. In addition to, excessive generation of ROS (reactive oxygen species) induced by various stimuli and which exceeds the antioxidant capacity of the organism leads to a diversity of pathophysiological processes such as inflammation, diabetes, genotoxicity and cancer ${ }^{30}$. In this paper, in order to define antioxidant activity of the synthesized Mannich Bases were researched different antioxidant method; iron binding effect, reducing power and 1,1-diphenyl-2-picryl-hydrazyl (DPPH) free radical scavenging activity ${ }^{31}$. Furthermore, the antimicrobial activities of novel Mannich bases were investigated with agar well diffusion method ${ }^{32}$. In the present paper, the starting materials (1a-g) were synthesized from the reactions of the corresponding ester ethoxycarbonylhydrazones with an aqueous solution of hydrazine hydrate ${ }^{33,34}$ and di-[2-(3-alkyl/aryl-4,5-dihydro- $1 \mathrm{H}-1,2,4$-triazol5-one-4-yl)-azomethinephenyl] isophtalates (2a-g) were obtained by the reactions of compounds (1a-g) with di-(2-formylphenyl) isophtalate. Then, the 
compounds 2 reacted with formaldehyde and morpholine to afford di-\{2-[1(morpholine-4-yl-methyl)-3-alkyl(aryl)-4,5-dihydro-1 $H$-1,2,4-triazol-5-one4-yl]-azomethinephenyl\} isophtalates (3a-g) (Scheme 1).

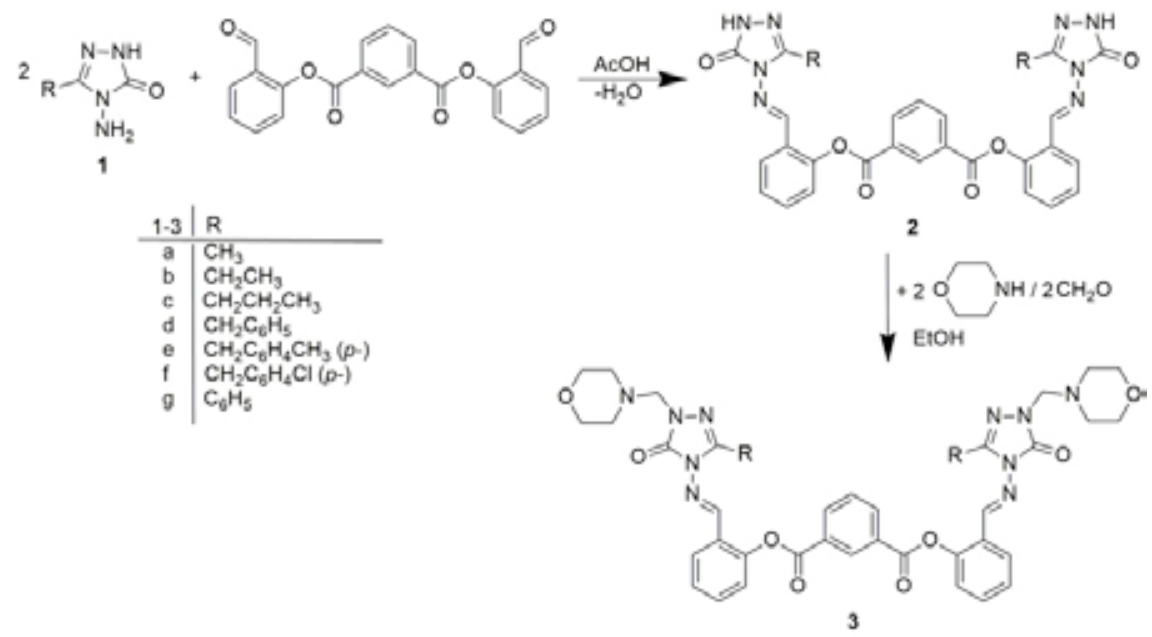

Scheme 1. The synthetic pathway of the compounds $\mathbf{2}$ and $\mathbf{3 .}$

\section{METHODOLOGY}

\section{Synthesis}

Preparation of Compounds 2a-g: 3-Alkyl/Aryl-4-amino-4,5-dihydro- $1 H$ 1,2,4-triazol-5-one (1) (0.01 mol) was dissolved in acetic acid (20 mL) and treated with di-(2-formylphenyl) isophtalate (0.01 mol). The mixture was refluxed for 1.5 hours and then evaporated at $50-55^{\circ} \mathrm{C}$ in vacuo. Several recrystallizations of the residue from ethanol gave pure compound di-[2-(3-alkyl/ aryl-4,5-dihydro-1H-1,2,4-triazol-5-one-4-yl)-azomethinephenyl] isophtalate (2) as white colour crystals.

Preparation of Compounds $\mathbf{3 a - g}$ : Compound 2 (5 mmol) was dissolved absolute ethanol and to this solution were added to formaldehyde (\% 37, $10 \mathrm{mmol}$ ) and morpholine $(6 \mathrm{mmol})$. The reaction mixture was refluxed for 4 hours and filtered. The crude product was recrystallized from ethanol.

Physical data of the new compounds (2a-g and $\mathbf{3 a - g}$ ) are presented in Table $\mathbf{1}$. IR, ${ }^{1} \mathrm{H}-\mathrm{NMR}$ and ${ }^{13} \mathrm{C}-\mathrm{NMR}$ spectral data are given in Tables 2-6. 
Table 1. Physical data of the compounds $\mathbf{2}$ and $\mathbf{3}$

\begin{tabular}{c|c|c|c|c|c|c|c|c|c|c|c|c|c|c}
\hline Compounds & 2a & 2b & 2c & 2d & 2e & 2f & 2g & 3a & 3b & 3c & 3d & 3e & 3f & 3g \\
\hline $\begin{array}{c}\text { \% Yield } \\
99\end{array}$ & 98 & 90 & 96 & 97 & 96 & 90 & 92 & 91 & 91 & 94 & 90 & 92 & 90 \\
\hline $\begin{array}{c}\text { Melting } \\
\text { Point }\left({ }^{\circ} \mathrm{C}\right)\end{array}$ & 271 & 233 & 226 & 273 & 260 & 243 & 251 & 224 & 183 & 165 & 176 & 158 & 155 & 206 \\
\hline
\end{tabular}

Table 2. IR data of the compounds $\mathbf{2}$ and $\mathbf{3}\left(\mathrm{cm}^{-1}\right)$.

\begin{tabular}{|c|c|c|c|c|c|c|c|c|}
\hline Compounds & $\mathrm{n}_{\mathrm{NH}}$ & $\mathrm{n}_{\mathrm{C}=0}$ & $\mathrm{n}_{\mathrm{C}=\mathrm{N}}$ & $\mathrm{n}_{\mathrm{co0}}$ & $\begin{array}{c}\mathbf{n}_{1,4-\text { disub- }} \\
\text { stituted } \\
\text { benzenoid } \\
\quad \text { ring } \\
\end{array}$ & $\begin{array}{c}\mathrm{n}_{1,3-} \\
\text { disubstituted } \\
\text { benzenoid } \\
\text { ring } \\
\end{array}$ & $\begin{array}{c}\mathbf{n}_{1,2-\text {-disub- }} \\
\text { stituted } \\
\text { benzenoid } \\
\text { ring } \\
\end{array}$ & $\begin{array}{c}\mathbf{n}_{\text {monosub- }} \\
\text { stituted } \\
\text { benzenoid } \\
\text { ring } \\
\end{array}$ \\
\hline $2 a$ & 3191 & $\begin{array}{l}1744, \\
1713\end{array}$ & 1604 & 1208 & - & $\begin{array}{l}871 \\
\text { and } \\
789\end{array}$ & 754 & - \\
\hline $2 b$ & 3188 & $\begin{array}{l}1739, \\
1705\end{array}$ & 1598 & 1203 & - & $\begin{array}{l}902 \\
\text { and } \\
800\end{array}$ & 761 & - \\
\hline $2 c$ & 3180 & 1710 & 1598 & 1207 & - & $\begin{array}{l}902 \\
\text { and } \\
824\end{array}$ & 752 & - \\
\hline $2 d$ & 3183 & 1709 & 1596 & 1202 & - & $\begin{array}{l}904 \\
\text { and } \\
819\end{array}$ & 752 & $\begin{array}{l}752 \\
\text { and } \\
694\end{array}$ \\
\hline $2 e$ & 3173 & $\begin{array}{l}1745, \\
1705\end{array}$ & 1598 & 1200 & 829 & $\begin{array}{l}903 \\
\text { and } \\
796\end{array}$ & 755 & - \\
\hline $2 f$ & 3185 & $\begin{array}{l}1740 \\
1705\end{array}$ & 1598 & 1201 & 821 & $\begin{array}{l}903 \\
\text { and } \\
792\end{array}$ & 749 & - \\
\hline $2 g$ & 3180 & 1705 & 1603 & 1203 & - & $\begin{array}{l}905 \\
\text { and } \\
802\end{array}$ & 756 & - \\
\hline $3 a$ & - & $\begin{array}{l}1742, \\
1704\end{array}$ & 1597 & 1215 & - & $\begin{array}{l}856 \\
\text { and } \\
768\end{array}$ & 768 & - \\
\hline $3 b$ & - & $\begin{array}{l}1742 \\
1700\end{array}$ & 1593 & 1204 & - & $\begin{array}{l}897 \\
\text { and } \\
765\end{array}$ & 765 & - \\
\hline $3 c$ & - & $\begin{array}{l}1744 \\
1700\end{array}$ & 1591 & 1205 & - & $\begin{array}{l}897 \\
\text { and } \\
765\end{array}$ & 757 & - \\
\hline $3 d$ & - & $\begin{array}{l}1742 \\
1700\end{array}$ & 1590 & 1207 & - & $\begin{array}{l}904 \\
\text { and } \\
762\end{array}$ & 762 & $\begin{array}{l}762 \\
\text { and } \\
709\end{array}$ \\
\hline $3 e$ & - & $\begin{array}{l}1746 \\
1701\end{array}$ & 1596 & 1206 & 841 & $\begin{array}{l}904 \\
\text { and } \\
790\end{array}$ & 757 & - \\
\hline
\end{tabular}




\begin{tabular}{c|c|c|c|c|c|c|c|c}
\hline $3 f$ & - & $\begin{array}{l}1744, \\
1701\end{array}$ & 1598 & 1211 & 820 & $\begin{array}{l}907 \\
\text { and } \\
801\end{array}$ & 745 & - \\
\hline $3 g$ & - & $\begin{array}{l}1740, \\
1697\end{array}$ & 1604 & 1207 & - & $\begin{array}{l}896 \\
\text { and } \\
800\end{array}$ & 766 & $\begin{array}{l}766 \\
\text { and } \\
687\end{array}$ \\
\hline
\end{tabular}

Table 3. ${ }^{1} \mathrm{H}-\mathrm{NMR}$ data of the compounds 2 (DMSO- $\left.d_{6}, \delta / p p m\right)$

\begin{tabular}{|c|c|c|c|c|c|c|c|}
\hline Compounds & $2 \mathrm{CH}_{3}$ & $2 \mathrm{CH}_{2}$ & $2 \mathrm{PhCH}_{3}$ & $2 \mathrm{CH}_{2}$ & Aromatic $\mathrm{H}$ & $2 \mathrm{~N}=\mathrm{CH}$ & $2 \mathrm{NH}$ \\
\hline $2 \mathrm{a}$ & $2.10(s)$ & - & - & - & $\begin{array}{c}\text { 7.48-7.53(m,4H), } \\
7.66(\mathrm{td}, 2 \mathrm{H}, \mathrm{J}=8.00,1.60 \\
\mathrm{Hz}), 7.90(\mathrm{t}, 1 \mathrm{H}, \mathrm{J}=8.80 \mathrm{~Hz}), \\
8.04(\mathrm{dd}, 2 \mathrm{H}, \mathrm{J}=8.00 \mathrm{~Hz}), \\
8.54(\mathrm{dd}, 2 \mathrm{H}, \mathrm{J}=8.00,1.60 \\
\mathrm{Hz}), 8.84(\mathrm{t}, 1 \mathrm{H}, \mathrm{J}=1.60 \mathrm{~Hz})\end{array}$ & $9.93(\mathrm{~s})$ & $11.75(\mathrm{~s})$ \\
\hline $2 b$ & $\begin{array}{c}1.09(\mathrm{t}, \mathrm{J}= \\
7.60 \mathrm{~Hz})\end{array}$ & - & - & $\begin{array}{c}2.47(\mathrm{q}, \\
\mathrm{J}=7.60 \mathrm{~Hz})\end{array}$ & $\begin{array}{c}7.48-7.52(\mathrm{~m}, 4 \mathrm{H}), \\
7.66(\mathrm{td}, 2 \mathrm{H}, \mathrm{J}=8.40,1.60 \\
\mathrm{Hz}), 7.91(\mathrm{t}, \mathrm{H}, \mathrm{H}, \mathrm{J}=8.00 \mathrm{~Hz}), \\
8.02(\mathrm{~d}, 2 \mathrm{H}, \mathrm{J}=8.00 \mathrm{~Hz}) \\
8.55(\mathrm{dd}, 2 \mathrm{H}, \mathrm{J}=8.00,2.00 \mathrm{~Hz}) \\
8.85(\mathrm{~s}, 1 \mathrm{H})\end{array}$ & $9.93(\mathrm{~s})$ & $11.75(\mathrm{~s})$ \\
\hline $2 c$ & $\begin{array}{c}0.85(\mathrm{t}, \mathrm{J}= \\
7.20 \mathrm{~Hz})\end{array}$ & $\begin{array}{c}\text { 1.57(sext, } \\
\mathrm{J}=7.20 \mathrm{~Hz})\end{array}$ & - & $\begin{array}{l}2.43(\mathrm{t}, \mathrm{J}= \\
7.20 \mathrm{~Hz})\end{array}$ & $\begin{array}{c}7.49-7.53(\mathrm{~m}, 4 \mathrm{H}), \\
7.67(\mathrm{t}, 2 \mathrm{H}, \mathrm{J}=8.00 \mathrm{~Hz}), \\
7.91(\mathrm{t}, 1 \mathrm{H}, \mathrm{J}=8.00 \mathrm{~Hz}), \\
8.02(\mathrm{dd}, 2 \mathrm{H}, \mathrm{J}=8.00,1.20 \mathrm{~Hz}), \\
8.55(\mathrm{dd}, 2 \mathrm{H}, \mathrm{J}=8.00,1.60 \mathrm{~Hz}), \\
8.85(\mathrm{~s}, 1 \mathrm{H})\end{array}$ & $9.92(s)$ & $11.79(\mathrm{~s})$ \\
\hline $2 d$ & - & - & - & $3.92(\mathrm{~s})$ & $\begin{array}{c}\text { 7.19-7.31(m,10H), } \\
7.47-7.50(\mathrm{~m}, 4 \mathrm{H}), \\
7.65(\mathrm{td}, 2 \mathrm{H}, \mathrm{J}=8.00,1.60 \\
\mathrm{Hz}), 7.86(\mathrm{t}, 1 \mathrm{H}, \mathrm{J}=8.00 \mathrm{~Hz}), \\
7.99(\mathrm{~d}, 2 \mathrm{H}, \mathrm{J}=8.00 \mathrm{~Hz}) \\
8.51(\mathrm{dd}, 2 \mathrm{H}, \mathrm{J}=8.00,1.60 \mathrm{~Hz}), \\
8.82(\mathrm{~s}, 1 \mathrm{H})\end{array}$ & $9.91(\mathrm{~s})$ & $11.91(\mathrm{~s})$ \\
\hline $2 e$ & - & - & $2.23(s)$ & $3.86(\mathrm{~s})$ & $\begin{array}{c}7.08(\mathrm{~d}, 4 \mathrm{H}, \mathrm{J}=8.00 \mathrm{~Hz}), \\
7.14(\mathrm{~d}, 4 \mathrm{H}, \mathrm{J}=8.00 \mathrm{~Hz}), 7.46-7.51 \\
(\mathrm{~m}, 4 \mathrm{H}), 7.65(\mathrm{td}, 2 \mathrm{H}, \mathrm{J}=8.00,1.60 \\
\mathrm{Hz}), 7.86(\mathrm{t}, 1 \mathrm{H}, \mathrm{J}=8.00 \mathrm{~Hz}), \\
8.00(\mathrm{dd}, 2 \mathrm{H}, \mathrm{J}=7.60,1.20 \mathrm{~Hz}), \\
8.51(\mathrm{dd}, 2 \mathrm{H}, \mathrm{J}=8.00,1.60 \mathrm{~Hz}), \\
8.83(\mathrm{~s}, 1 \mathrm{H})\end{array}$ & $9.90(s)$ & $12.00(s)$ \\
\hline $2 f$ & - & - & - & $3.93(\mathrm{~s})$ & $\begin{array}{c}\text { 7.27(d, } 4 \mathrm{H}, \mathrm{J}=8.40 \mathrm{~Hz}), \\
7.35(\mathrm{~d}, 4 \mathrm{H}, \mathrm{J}=8.40 \mathrm{~Hz}), 7.47- \\
7.51(\mathrm{~m}, 4 \mathrm{H}), 7.66(\mathrm{t}, 2 \mathrm{H}, \mathrm{J}=8.00 \\
\mathrm{Hz}), 7.86(\mathrm{t}, 1 \mathrm{H}, \mathrm{J}=8.00 \mathrm{~Hz}), \\
7.99(\mathrm{~d}, 2 \mathrm{H}, \mathrm{J}=7.60 \mathrm{~Hz}), \\
8.51(\mathrm{dd}, 2 \mathrm{H}, \mathrm{J}=8.00,1.60 \mathrm{~Hz}), \\
8.82(\mathrm{~s}, 1 \mathrm{H})\end{array}$ & $9.92(s)$ & $11.93(\mathrm{~s})$ \\
\hline $2 g$ & - & - & - & - & $\begin{array}{c}7.48-7.55(\mathrm{~m}, 10 \mathrm{H}), \\
7.67-7.82(\mathrm{~m}, 7 \mathrm{H}), \\
7.99(\mathrm{dd}, 2 \mathrm{H}, \mathrm{J}=8.00,1.20 \mathrm{~Hz}), \\
8.40(\mathrm{dd}, 2 \mathrm{H}, \mathrm{J}=7.60,1.60 \mathrm{~Hz}), \\
8.68(\mathrm{~s}, 1 \mathrm{H})\end{array}$ & $9.88(s)$ & $12.32(s)$ \\
\hline
\end{tabular}


Table 4. ${ }^{13} \mathrm{C}$-NMR data of the compounds 2 (DMSO- $\left.d_{6}, \delta / p p m\right)$

\begin{tabular}{|c|c|c|c|c|c|c|c|}
\hline Comp. & $2 \mathrm{COO}$ & $\begin{array}{c}\text { 2Triazole } \\
C_{5}\end{array}$ & $2 \mathrm{~N}=\mathrm{CH}$ & $\begin{array}{c}\text { 2Triazole } \\
\mathrm{C}_{3} \\
\end{array}$ & Aromatic C & $\begin{array}{c}\text { C3-Aromatik } \\
\text { C }\end{array}$ & Aliphatic C \\
\hline $2 a$ & 163.54 & 149.51 & 148.91 & 144.09 & $\begin{array}{c}123.64(2 \mathrm{CH}), \\
126.01(2 \mathrm{C}), \\
126.95(2 \mathrm{CH}), \\
127.70(2 \mathrm{CH}), \\
129.42(2 \mathrm{C}), \\
130.16(\mathrm{CH}), \\
131.00(\mathrm{CH}), \\
132.54(2 \mathrm{CH}), \\
135.22(2 \mathrm{CH}), \\
151.16(2 \mathrm{C})\end{array}$ & - & $10.85\left(2 \mathrm{CH}_{3}\right)$ \\
\hline $2 b$ & 163.54 & 149.46 & 149.09 & 147.86 & $\begin{array}{c}123.66(2 \mathrm{CH}), \\
126.03(2 \mathrm{C}), \\
126.98(2 \mathrm{CH}), \\
127.86(2 \mathrm{CH}), \\
129.42(2 \mathrm{C}), \\
130.14(\mathrm{CH}), \\
131.01(\mathrm{CH}), \\
132.53(2 \mathrm{CH}), \\
135.21(2 \mathrm{CH}), \\
151.30(2 \mathrm{C})\end{array}$ & - & $\begin{array}{l}9.87\left(2 \mathrm{CH}_{2} \mathrm{CH}_{3}\right), \\
18.31\left(2 \mathrm{CH}_{2} \mathrm{CH}_{3}\right)\end{array}$ \\
\hline $2 c$ & 163.53 & 149.45 & 149.23 & 146.71 & $\begin{array}{c}123.64(2 \mathrm{CH}), \\
126.03(2 \mathrm{C}), \\
127.00(2 \mathrm{CH}), \\
127.87(2 \mathrm{CH}), \\
129.44(2 \mathrm{C}), \\
130.19(\mathrm{CH}), \\
131.02(\mathrm{CH}), \\
132.54(2 \mathrm{CH}), \\
135.21(2 \mathrm{CH}), \\
151.23(2 \mathrm{C})\end{array}$ & - & $\begin{array}{c}13.31\left(2 \mathrm{CH}_{2} \mathrm{CH}_{2} \mathrm{CH}_{3}\right), \\
18.65 \\
\left(2 \mathrm{CH}_{2} \mathrm{CH}_{2} \mathrm{CH}_{3}\right), \\
26.46\left(2 \mathrm{CH}_{2} \mathrm{CH}_{2} \mathrm{CH}_{3}\right)\end{array}$ \\
\hline $2 d$ & 163.51 & 149.68 & 148.46 & 146.09 & $\begin{array}{c}123.54(2 \mathrm{CH}), \\
125.97(2 \mathrm{C}), \\
126.67(2 \mathrm{CH}), \\
127.12(2 \mathrm{CH}), \\
129.32(2 \mathrm{C}), \\
130.13(\mathrm{CH}), \\
131.03(\mathrm{CH}), \\
132.59(2 \mathrm{CH}), \\
135.20(2 \mathrm{CH}), \\
151.10(2 \mathrm{C})\end{array}$ & $\begin{array}{c}126.94(2 \mathrm{CH}), \\
\text { 128.37(4CH), } \\
\text { 128.72(4CH), } \\
135.58(2 \mathrm{C})\end{array}$ & $30.82\left(2 \mathrm{CH}_{2} \mathrm{Ph}\right)$ \\
\hline $2 e$ & 163.50 & 149.66 & 148.43 & 146.23 & $\begin{array}{c}123.52(2 \mathrm{CH}), \\
125.98(2 \mathrm{C}), \\
126.93(2 \mathrm{CH}), \\
127.14(2 \mathrm{CH}), \\
129.32(2 \mathrm{C}), \\
130.11(\mathrm{CH}), \\
131.03(\mathrm{CH}), \\
132.56(2 \mathrm{CH}), \\
135.18(2 \mathrm{CH}), \\
151.17(2 \mathrm{C})\end{array}$ & $\begin{array}{c}128.58(4 \mathrm{CH}), \\
128.94(4 \mathrm{CH}), \\
132.46(2 \mathrm{C}) \\
135.74(2 \mathrm{C})\end{array}$ & $\begin{array}{l}20.55\left(2 \mathrm{PhCH}_{3}\right) \\
30.43\left(2 \mathrm{CH}_{2} \mathrm{Ph}\right)\end{array}$ \\
\hline
\end{tabular}




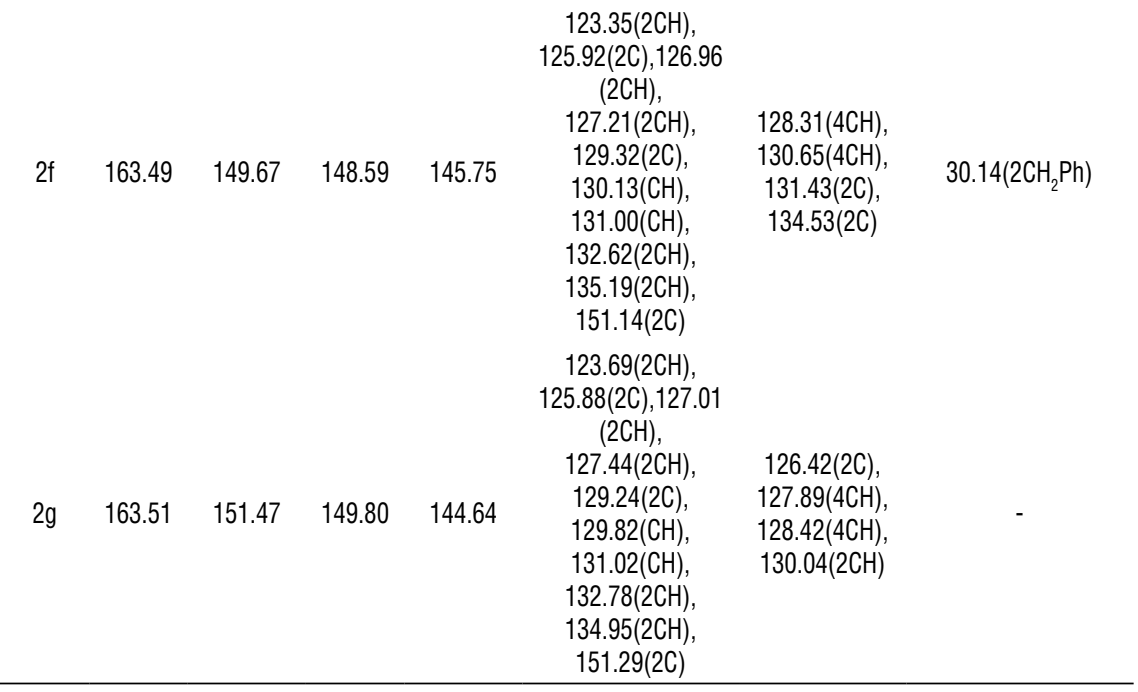

Table 5. ${ }^{1} \mathrm{H}-\mathrm{NMR}$ data of the compounds 3 (DMSO- $\left.d_{6}, \delta / p p m\right)$

\begin{tabular}{|c|c|c|c|c|c|c|c|c|}
\hline Comp. & $2 \mathrm{CH}_{3}$ & $2 \mathrm{CH}_{2}$ & $2 \mathrm{CH}_{2}$ & $2 \mathrm{CH}_{2} \mathrm{NCH}_{2}$ & $2 \mathrm{CH}_{2} \mathrm{OCH}_{2}$ & $2 \mathrm{NCH}_{2} \mathrm{~N}$ & Aromatic $\mathrm{H}$ & $2 \mathrm{~N}=\mathrm{CH}$ \\
\hline $3 a$ & $2.11(\mathrm{~s})$ & - & - & $2.54(\mathrm{~m})$ & $3.48(\mathrm{~m})$ & $4.38(s)$ & $\begin{array}{c}7.49-7.53(\mathrm{~m}, 4 \mathrm{H}), \\
7.67(\mathrm{td}, 2 \mathrm{H}, \\
\mathrm{J}=8.40,1.60 \mathrm{~Hz}) \\
7.91(\mathrm{t}, 1 \mathrm{H}, \mathrm{J}=8.00 \mathrm{~Hz}), \\
8.05(\mathrm{~d}, 2 \mathrm{H}, \mathrm{J}=7.60 \mathrm{~Hz}) \\
8.54(\mathrm{dd}, 2 \mathrm{H}, \mathrm{J}=8.00,1.60 \\
\mathrm{Hz}), 8.87(\mathrm{~s}, 1 \mathrm{H})\end{array}$ & $\begin{array}{l}9.93 \\
(\mathrm{~s})\end{array}$ \\
\hline $3 b$ & $\begin{array}{c}1.09(\mathrm{t}, \\
\mathrm{J}=7.60 \\
\mathrm{~Hz})\end{array}$ & $\begin{array}{l}2.50(q, J= \\
7.60 \mathrm{~Hz})\end{array}$ & - & $2.55(\mathrm{~m})$ & $3.49(\mathrm{~m})$ & $4.40(s)$ & $\begin{array}{c}7.49-7.53(\mathrm{~m}, 4 \mathrm{H}), \\
7.67(\mathrm{t}, 2 \mathrm{H}, \mathrm{J}=8.00 \mathrm{~Hz}), \\
7.92(\mathrm{t}, 1 \mathrm{H}, \mathrm{J}=8.00 \mathrm{~Hz}), \\
8.03(\mathrm{~d}, 2 \mathrm{H}, \mathrm{J}=7.60 \\
\mathrm{Hz}), 8.55(\mathrm{~d}, 2 \mathrm{H}, \mathrm{J} \\
=7.60 \mathrm{~Hz}), 8.87(\mathrm{~s}, 1 \mathrm{H})\end{array}$ & $\begin{array}{l}9.92 \\
(\mathrm{~s})\end{array}$ \\
\hline $3 c$ & $\begin{array}{c}0.85(\mathrm{t}, \\
\mathrm{J}=7.20 \\
\mathrm{~Hz})\end{array}$ & $\begin{array}{c}1.57 \\
(\text { sext, } \mathrm{J}= \\
7.20 \mathrm{~Hz})\end{array}$ & $\begin{array}{c}2.48(\mathrm{t}, \mathrm{J} \\
=7.20 \\
\mathrm{~Hz})\end{array}$ & $2.55(\mathrm{~m})$ & $\begin{array}{c}3.49(\mathrm{t}, \mathrm{J}= \\
4.40 \mathrm{~Hz})\end{array}$ & $4.41(\mathrm{~s})$ & $\begin{array}{c}\text { 7.50-7.53(m,4H), } \\
7.65-7.68(\mathrm{~m}, 2 \mathrm{H}), \\
7.92(\mathrm{t}, 1 \mathrm{H}, \mathrm{J}=8.00 \mathrm{~Hz}), \\
8.00-8.05(\mathrm{~m}, 2 \mathrm{H}), \\
8.54(\mathrm{dd}, 2 \mathrm{H}, \mathrm{J}=8.00,1.60 \\
\mathrm{Hz}), \\
8.87(\mathrm{~d}, 1 \mathrm{H}, \mathrm{J}=1.60 \mathrm{~Hz})\end{array}$ & $\begin{array}{l}9.92 \\
(s)\end{array}$ \\
\hline $3 d$ & - & - & $3.95(\mathrm{~s})$ & $\begin{array}{c}2.48(\mathrm{t}, \mathrm{J}= \\
4.40 \mathrm{~Hz})\end{array}$ & $3.47(\mathrm{~m})$ & $4.42(s)$ & $\begin{array}{c}7.18-7.31(\mathrm{~m}, 10 \mathrm{H}), \\
7.47-7.51(\mathrm{~m}, 4 \mathrm{H}), \\
7.66(\mathrm{td}, 2 \mathrm{H}, \mathrm{J}=7.60,1.20 \\
\mathrm{Hz}), \\
7.85(\mathrm{t}, 1 \mathrm{H}, \mathrm{J}=8.00 \mathrm{~Hz}), \\
7.99(\mathrm{~d}, 2 \mathrm{H}, \mathrm{J}=7.60 \mathrm{~Hz}), \\
8.49(\mathrm{~d}, 2 \mathrm{H}, \mathrm{J}=7.60,1.60 \\
\mathrm{Hz}), 8.83(\mathrm{~s}, 1 \mathrm{H})\end{array}$ & $\begin{array}{l}9.89 \\
(\mathrm{~s})\end{array}$ \\
\hline
\end{tabular}




\begin{tabular}{|c|c|c|c|c|c|c|c|c|}
\hline $3 e$ & $\begin{array}{l}2.22 \\
(s)\end{array}$ & & $3.90(\mathrm{~s})$ & $\begin{array}{c}2.46(\mathrm{t}, \mathrm{J}= \\
4.40 \mathrm{~Hz})\end{array}$ & $\begin{array}{c}3.47(\mathrm{t}, \mathrm{J}= \\
4.40 \mathrm{~Hz})\end{array}$ & $4.41(\mathrm{~s})$ & $\begin{array}{c}7.07-7.14(\mathrm{~m}, 8 \mathrm{H}), \\
7.46-7.51(\mathrm{~m}, 4 \mathrm{H}), \\
7.66(\mathrm{td}, 2 \mathrm{H}, \mathrm{J} \\
=7.60,1.60 \mathrm{~Hz}), \\
7.85(\mathrm{t}, 1 \mathrm{H}, \mathrm{J}=7.60 \mathrm{~Hz}), \\
8.00(\mathrm{dd}, 2 \mathrm{H}, \mathrm{J}=8.00,1.60 \\
\mathrm{Hz}), \\
8.49(\mathrm{dd}, 2 \mathrm{H}, \mathrm{J}=8.00,1.60 \\
\mathrm{Hz}), 8.83(\mathrm{~m}, 1 \mathrm{H})\end{array}$ & $\begin{array}{l}9.88 \\
(\mathrm{~s})\end{array}$ \\
\hline $3 f$ & - & - & $3.96(\mathrm{~s})$ & $\begin{array}{c}2.46(\mathrm{t}, \mathrm{J}= \\
4.40 \mathrm{~Hz})\end{array}$ & $\begin{array}{c}3.47(\mathrm{t}, \mathrm{J}= \\
4.40 \mathrm{~Hz})\end{array}$ & $4.41(\mathrm{~s})$ & $\begin{array}{c}7.27(\mathrm{~d}, 4 \mathrm{H}, \mathrm{J}=8.40 \mathrm{~Hz}), \\
7.35(\mathrm{~d}, 4 \mathrm{H}, \mathrm{J}=8.40 \\
\mathrm{Hz}), 7.47-7.49(\mathrm{~m}, 4 \mathrm{H}), \\
7.64-7.69(\mathrm{~m}, 2 \mathrm{H}) \\
7.85(\mathrm{t}, 1 \mathrm{H}, \mathrm{J}=8.00 \mathrm{~Hz}), \\
7.97-8.01(\mathrm{~m}, 2 \mathrm{H}), \\
8.49(\mathrm{dd}, 2 \mathrm{H}, \mathrm{J}=7.60, \\
1.60 \mathrm{~Hz}), 8.83(\mathrm{~m}, 1 \mathrm{H})\end{array}$ & $\begin{array}{l}9.91 \\
(\mathrm{~s})\end{array}$ \\
\hline $3 g$ & - & - & - & $2.54(\mathrm{~m})$ & $\begin{array}{c}3.49(\mathrm{t}, \mathrm{J}= \\
4.40 \mathrm{~Hz})\end{array}$ & $4.52(\mathrm{~s})$ & $\begin{array}{c}7.47-7.55(\mathrm{~m}, 12 \mathrm{H}), 7.70 \\
(\mathrm{td}, 2 \mathrm{H}, \mathrm{J}=8.40,1.60 \\
\mathrm{Hz}), 7.76-7.82(\mathrm{~m}, 3 \mathrm{H}), \\
7.99(\mathrm{dd}, 2 \mathrm{H}, \mathrm{J}=8.00,1.60 \\
\mathrm{Hz}), \\
8.38(\mathrm{dd}, 2 \mathrm{H}, \mathrm{J}=8.00,1.60 \\
\mathrm{Hz}), 8.71(\mathrm{~m}, 1 \mathrm{H})\end{array}$ & $9.88(s)$ \\
\hline
\end{tabular}

Table $6 .{ }^{13} \mathrm{C}$-NMR data of the compounds 3 (DMSO- $\left.d_{6}, \delta / p p m\right)$

\begin{tabular}{|c|c|c|c|c|c|c|c|c|c|c|}
\hline Comp. & $2 \mathrm{COO}$ & a & $2 \mathrm{~N}=\mathrm{CH}$ & b & Aromatic C & $\begin{array}{c}\text { C3- } \\
\text { Aromatik C }\end{array}$ & c & d & e & Aliphatic C \\
\hline $3 a$ & 163.53 & 149.53 & 149.18 & 142.97 & $\begin{array}{c}123.66(2 \mathrm{CH}) \text {, } \\
125.84(2 \mathrm{C}), \\
126.97(2 \mathrm{CH}), \\
127.90(2 \mathrm{CH}), \\
129.48(2 \mathrm{C}), \\
130.23(\mathrm{CH}), \\
130.97(\mathrm{CH}), \\
132.67(2 \mathrm{CH}), \\
135.29(2 \mathrm{CH}), \\
150.15(2 \mathrm{C})\end{array}$ & - & 65.96 & 65.79 & 49.86 & $10.81\left(2 \mathrm{CH}_{3}\right)$ \\
\hline $3 b$ & 163.53 & 149.50 & 149.35 & 146.68 & $\begin{array}{c}123.67(2 \mathrm{CH}), \\
125.86(2 \mathrm{C}), \\
127.00(2 \mathrm{CH}), \\
127.99(2 \mathrm{CH}), \\
129.48(2 \mathrm{C}), \\
130.21(\mathrm{CH}), \\
130.97(\mathrm{CH}), \\
132.67(2 \mathrm{CH}), \\
135.28(2 \mathrm{CH}), \\
150.28(2 \mathrm{C})\end{array}$ & - & 65.96 & 65.82 & 49.88 & $\begin{array}{l}9.88\left(2 \mathrm{CH}_{2} \mathrm{CH}_{3}\right), \\
18.20\left(2 \mathrm{CH}_{2} \mathrm{CH}_{3}\right)\end{array}$ \\
\hline
\end{tabular}




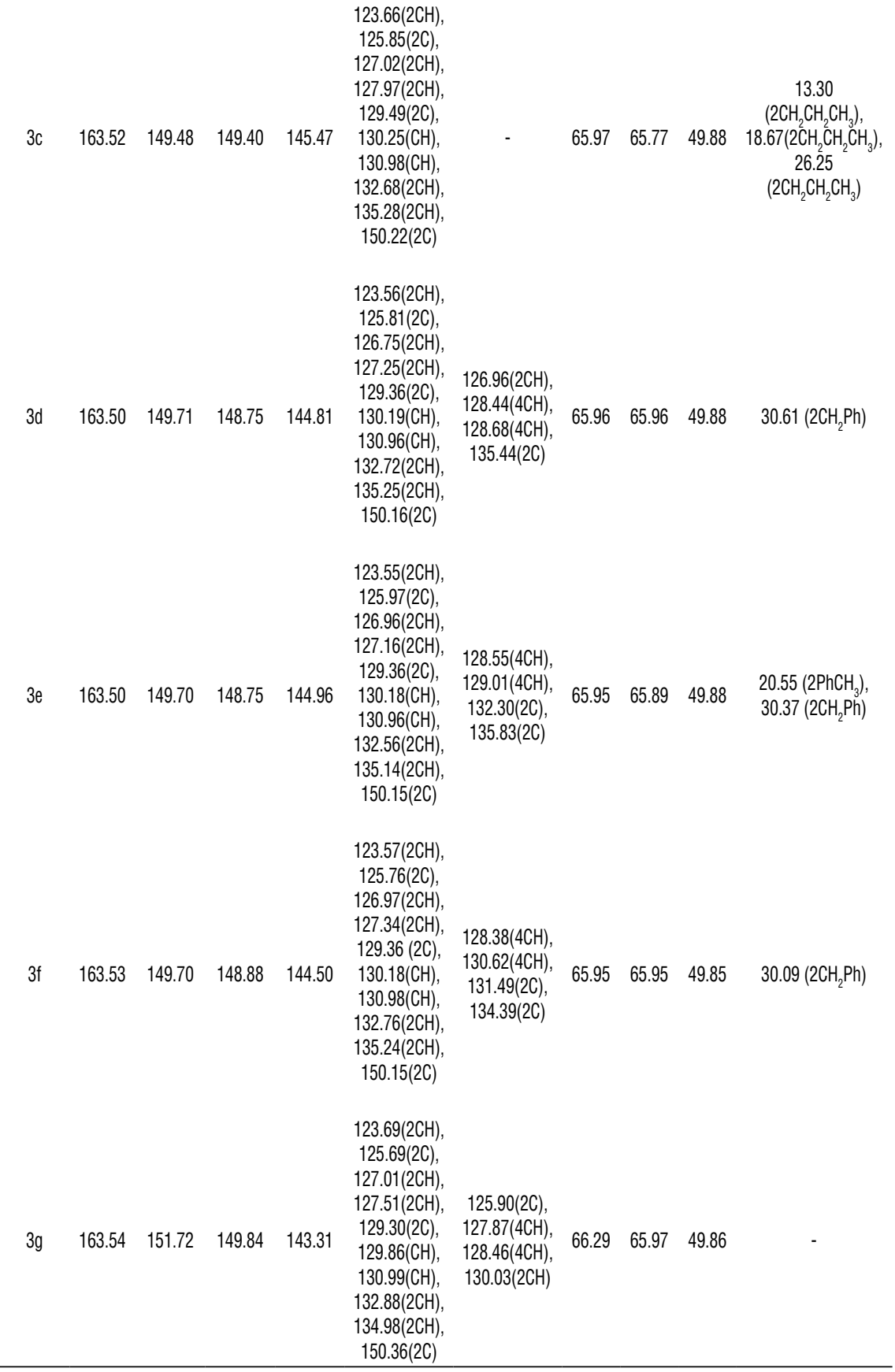

a) 2 Triazole $\mathrm{C}_{5}$, b) 2 Triazole $\mathrm{C}_{3}$, c) $2 \mathrm{CH}_{2} \mathrm{OCH}_{2}$, d) $2 \mathrm{NCH}_{2} \mathrm{~N}$, e) $2 \mathrm{CH}_{2} \mathrm{NCH}_{2}$ 


\section{Biological Methods}

\section{Antioxidant Activity}

Trichloroacetic acid (TCA), a-tocopherol, butylated hydroxyanisole (BHA), ethylenediaminetetraacetic acid (EDTA), butylated hydroxytoluene (BHT), 1,1-diphenyl-2-picryl-hydrazyl (DPPH), 3-(2-pyridyl)-5,6-bis(phenylsulfonic acid)-1,2,4-triazine (ferrozine) and ferrous chloride, were acquired from E. Merck. and Sigma-Aldrich.

\section{Reducing Power}

The reducing power of the compounds $\mathbf{2 a - g}$ and $\mathbf{3 a - g}$ were determined using the method of $\mathrm{Oyaizu}^{35}$ as explained in the literature ${ }^{31}$ and were shown in Table 8.

Table 7. The reducing power method

\begin{tabular}{|c|c|c|c|c|c|c|c|}
\hline Reagents & $S_{1}$ & $\mathrm{~S}_{2}$ & $\mathrm{~S}_{3}$ & $\mathrm{~N}_{1}$ & $\mathrm{~N}_{2}$ & $\mathrm{~N}_{3}$ & Blank \\
\hline Compound & - & - & - & $100 \mu \mathrm{L}$ & $250 \mu \mathrm{L}$ & $500 \mu \mathrm{L}$ & - \\
\hline Standard & $100 \mu \mathrm{L}$ & $250 \mu \mathrm{L}$ & $500 \mu \mathrm{L}$ & - & - & - & - \\
\hline Phosphate Buffer & $2,4 \mathrm{~mL}$ & $2,25 \mathrm{~mL}$ & $2,0 \mathrm{~mL}$ & $2,4 \mathrm{~mL}$ & $2,25 \mathrm{~mL}$ & $2,0 \mathrm{~mL}$ & - \\
\hline $\mathrm{K}_{3} \mathrm{Fe}(\mathrm{CN})_{6}$ & $2,5 \mathrm{~mL}$ & $2,5 \mathrm{~mL}$ & $2,5 \mathrm{~mL}$ & $2,5 \mathrm{~mL}$ & $2,5 \mathrm{~mL}$ & $2,5 \mathrm{~mL}$ & - \\
\hline
\end{tabular}

\section{Free Radical Scavenging Activity}

Free radical scavenging effect of the compounds $\mathbf{2 a - g}$ and $\mathbf{3 a - g}$ were estimated by DPPH', by the method of Blois ${ }^{36}$ as explained in the literature ${ }^{31}$ and were summarized in Table 8.

Table 8. The free radical scavenging effect method

\begin{tabular}{lcccccccc} 
Reagents & $\mathbf{S}_{1}$ & $\mathbf{S}_{2}$ & $\mathbf{S}_{3}$ & $\mathbf{N}_{1}$ & $\mathbf{N}_{2}$ & $\mathbf{N}_{3}$ & Blank & Control \\
\hline Compound & - & - & - & $50 \mu \mathrm{L}$ & $100 \mu \mathrm{L}$ & $150 \mu \mathrm{L}$ & - & - \\
Standard & $50 \mu \mathrm{L}$ & $100 \mu \mathrm{L}$ & $150 \mu \mathrm{L}$ & - & - & - & - & - \\
Ethyl Alcohol & $2,95 \mathrm{~mL}$ & $2,90 \mathrm{~mL}$ & $2,85 \mathrm{~mL}$ & $2,95 \mathrm{~mL}$ & $2,90 \mathrm{~mL}$ & $2,85 \mathrm{~mL}$ & - & $3 \mathrm{~mL}$ \\
DPPH & $1 \mathrm{~mL}$ & $1 \mathrm{~mL}$ & $1 \mathrm{~mL}$ & $1 \mathrm{~mL}$ & $1 \mathrm{~mL}$ & $1 \mathrm{~mL}$ & $4 \mathrm{~mL}$ & $1 \mathrm{~mL}$ \\
\hline
\end{tabular}

\section{Metal Chelating Activity}

The chelating of ferrous ions by the compounds $\mathbf{2 a - g}$ and $\mathbf{3 a - g}$ and references were measured according to the method of Dinis et al. ${ }^{37}$ as explained in the literature ${ }^{31}$ (Table 9). 
Table 9. The metal chelating activity method

\begin{tabular}{lcccccccc} 
Reagents & $\mathbf{S}_{1}$ & $\mathbf{S}_{2}$ & $\mathbf{S}_{3}$ & $\mathbf{N}_{1}$ & $\mathbf{N}_{2}$ & $\mathbf{N}_{3}$ & Blank & Control \\
\hline Compound & - & - & - & $30 \mu \mathrm{L}$ & $60 \mu \mathrm{L}$ & $90 \mu \mathrm{L}$ & - & - \\
Standard & $30 \mu \mathrm{L}$ & $60 \mu \mathrm{L}$ & $90 \mu \mathrm{L}$ & - & - & - & - & - \\
Ethyl Alcohol & $3,75 \mathrm{~mL}$ & $3,75 \mathrm{~mL}$ & $3,75 \mathrm{~mL}$ & $3,75 \mathrm{~mL}$ & $3,75 \mathrm{~mL}$ & $3,75 \mathrm{~mL}$ & $3,75 \mathrm{~mL}$ & $3,75 \mathrm{~mL}$ \\
FeCl $_{2} 4 \mathrm{H}_{2} \mathbf{O}$ & $0,05 \mathrm{~mL}$ & $0,05 \mathrm{~mL}$ & $0,05 \mathrm{~mL}$ & $0,05 \mathrm{~mL}$ & $0,05 \mathrm{~mL}$ & $0,05 \mathrm{~mL}$ & $0,05 \mathrm{~mL}$ & $0,05 \mathrm{~mL}$ \\
Ferrozine & $0,2 \mathrm{~mL}$ & $0,2 \mathrm{~mL}$ & $0,2 \mathrm{~mL}$ & $0,2 \mathrm{~mL}$ & $0,2 \mathrm{~mL}$ & $0,2 \mathrm{~mL}$ & $0,2 \mathrm{~mL}$ & $0,2 \mathrm{~mL}$ \\
\hline
\end{tabular}

\section{Antimicrobial Activity}

Antimicrobial activities of $\mathbf{2 a - g}$ and $\mathbf{3 a - g}$ compounds were investigated simple susceptibility screening test using agar-well diffusion method ${ }^{22}$ as adapted earlier ${ }^{38}$. All microorganisms present in the test were provided from the Microbiologic Environmental Protection Laboratories Company in France. These microorganisms: Klebsiella pneumoniae ATCC4352, Pseudomonas aeruginosa ATCC27853, Escherichia coli ATCC259222, Staphylococcus aureus ATCC6538, Bacillus subtilis ATCC11774, Bacillus cereus ATCC11778.

\section{RESULTS AND DISCUSSION}

The synthesized seven new Schiff bases and seven new Mannich bases were identified using IR, ${ }^{1} \mathrm{H}-\mathrm{NMR},{ }^{13} \mathrm{C}-\mathrm{NMR}$ spectral data.

\section{Antioxidant Acivity}

In vitro antioxidant activities of fourteen new compounds $\mathbf{2 a - g}$ and $\mathbf{3} \mathbf{a}-\mathbf{g}$ were investigated. Antioxidant activities were determined by the methods showed below.

Total reductive capability using the potassium ferricyanide reduction method

The reducing power of the compounds $\mathbf{2 a - g}$ and $\mathbf{3 a - g}$ was determined as described in ${ }^{39,40}$. All compounds in different amounts showed lower absorption rates in this study than the standard compounds. Consequently, no activity was observed with respect to the reduction of metal ion complexes to their lower oxidation states or their involvement in an electron transfer reaction. In summary, synthesized compounds were not involved in reductive activities as seen in Figures $\mathbf{1}$ and $\mathbf{2}$. 


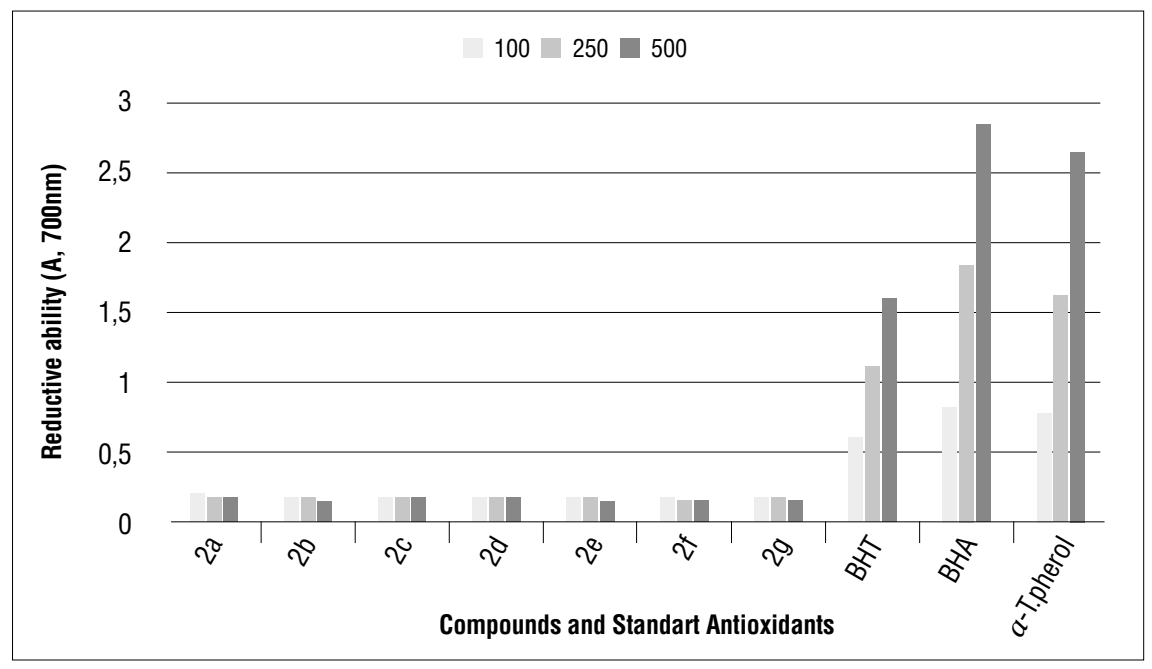

Figure 1. Total reductive potential of different concentrations of the compounds $2, \mathrm{BHT}, \mathrm{BHA}$ and $\alpha$-tocopherol.

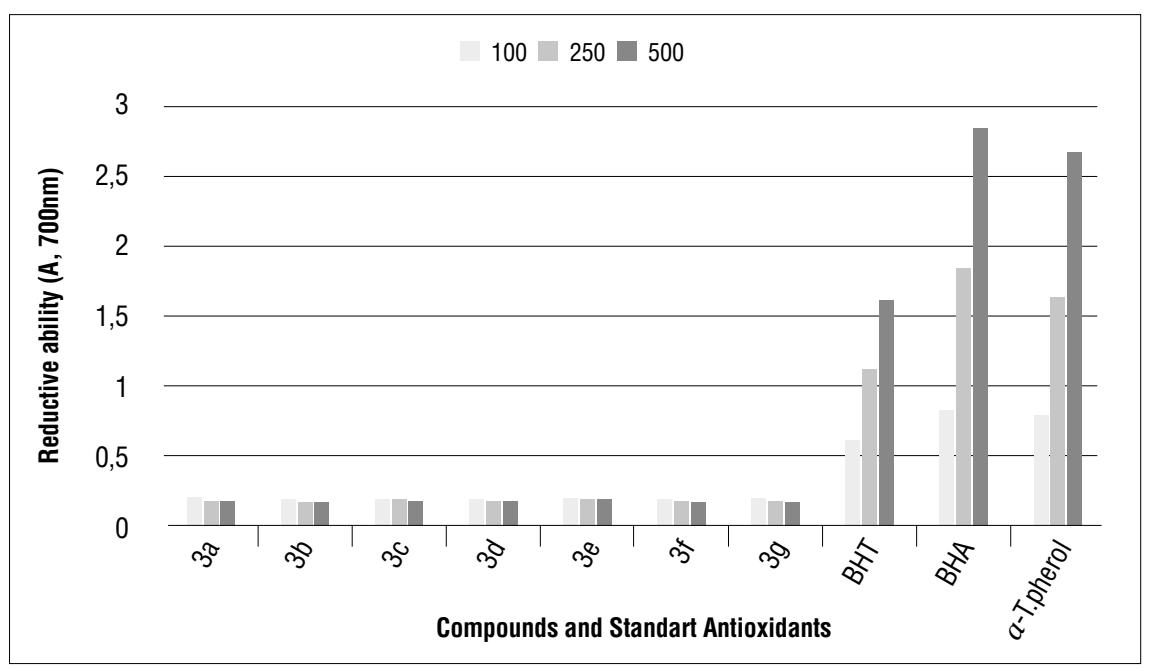

Figure 2. Total reductive potential of different concentrations of the compounds $3, \mathrm{BHT}, \mathrm{BHA}$ and $\alpha$-tocopherol. 


\section{DPPH· radical scavenging activity}

The scavenging effect of compounds 2a-g and 3a-g was estimated by DPPH as explained in ${ }^{41-43}$. The DPPH method was used to determine the antiradical activity of compounds and standard antioxidants such as BHA, BHT and a-tocopherol in the study. It has been found that recently synthesized compounds have no activity as radical scavengers as shown in Figures 3 and 4.

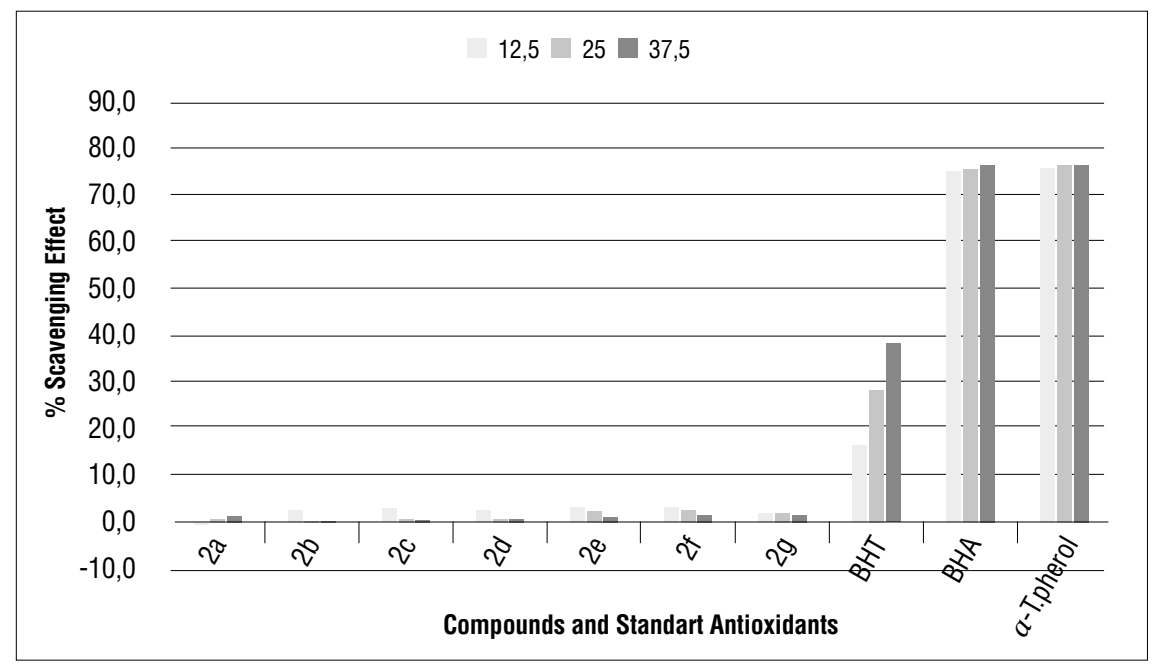

Figure 3. Scavenging effect of the compounds 2, BHT, BHA and $\alpha$-tocopherol at different concentrations (12.5-25-37.5 $\mu \mathrm{g} / \mathrm{mL})$.

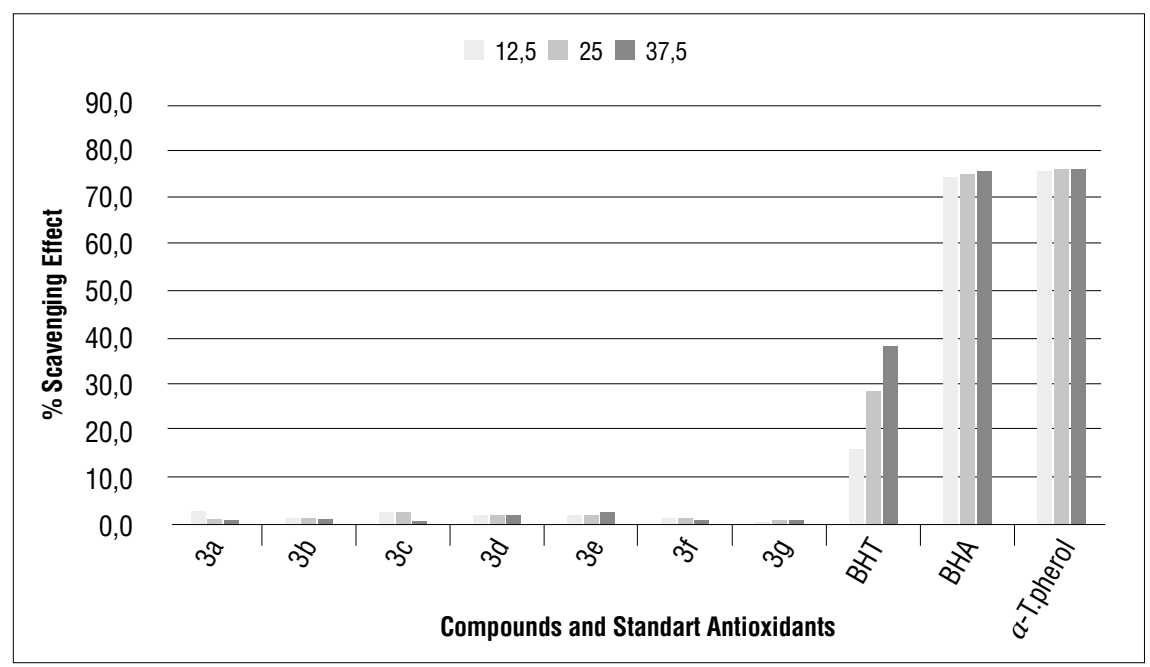

Figure 4. Scavenging effect of the compounds $3, \mathrm{BHT}, \mathrm{BHA}$ and $\alpha$-tocopherol at different concentrations $(12.5-25-37.5 \mu \mathrm{g} / \mathrm{mL})$. 


\section{Ferrous ion chelating activity}

The chelation effect against iron ions by the compounds and standards was determined. Ferrozin can form complexes quantitatively with $\mathrm{Fe}^{2+}$. In the presence of chelating agents, the complex formation is disturbed, so that the red colour of the complex decreases. The measurement of colour reduction thus allows the estimation of the chelating activity of the coexisting chelator ${ }^{44}$. Iron ion chelating activities of the compounds 2, 3, EDTA and $\alpha$-tocopherol are shown in Figures $\mathbf{5}$ and $\mathbf{6}$, respectively.

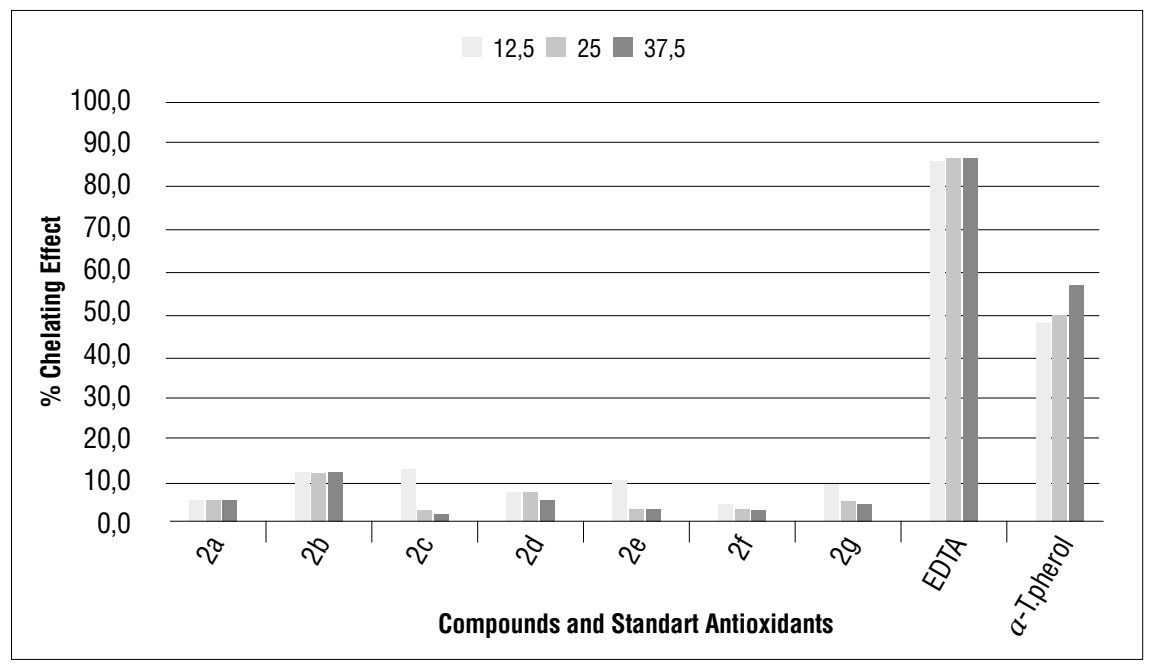

Figure 5. Metal chelating effect of different amount of the compounds 2, EDTA and $\alpha$-tocopherol on ferrous ions.

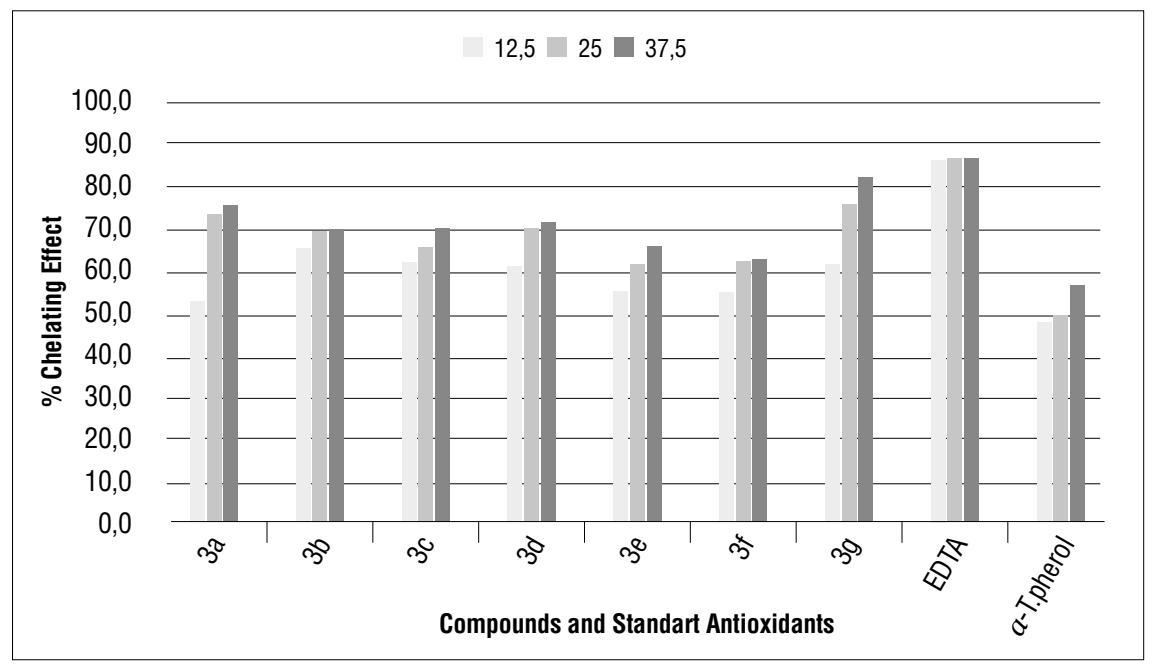

Figure 6. Metal chelating effect of different amount of the compounds 3, EDTA and $\alpha$-tocopherol on ferrous ions. 
The high metal-chelating activity refers to a low absorption level at $562 \mathrm{~nm}$. From the data in Figure 3, it can be deduced that the metal-chelating effects of compounds are concentration-dependent. As a result, compounds with significant iron-binding capacities can prove that their action as peroxidation inhibitors stems from their iron-binding capacities. The order of metal chelation of compounds and standards decreases when EDTA $>\mathbf{3 g}>\mathbf{3 a}>\mathbf{3 d}>\mathbf{3 b} \approx \mathbf{3 c}>$ 3e $>$ 3f $>$ a-tocopherol. Despite the low solubility rate for free iron, chelated iron complexes are known to have higher solubility rates in solutions that can be readily attributed to the ligand. Due to their potential involvement in ironcatalysed reactions, compound iron complexes could also be active.

\section{Antimicrobial Activity}

The antimicrobial activity of the compounds $\mathbf{2}$ and $\mathbf{3}$ were investigated. The results are shown in Table $\mathbf{1 0}$ and $\mathbf{1 1 .}$

Table 10. Zone diameters for antimicrobial activity of the $\mathbf{2 , 3}$ and Standart compounds

\begin{tabular}{|c|c|c|c|c|c|c|}
\hline \multirow{2}{*}{ Compound } & \multicolumn{6}{|c|}{ Microorganisms and Inhibition Zone (mm) } \\
\hline & K.p. & P.a. & E.c. & S.a. & B.s. & B.c. \\
\hline $2 a$ & - & - & - & - & - & - \\
\hline $2 b$ & - & - & - & - & - & - \\
\hline $2 c$ & - & - & - & - & - & - \\
\hline $2 d$ & - & - & - & - & - & - \\
\hline $2 e$ & - & - & - & - & - & - \\
\hline $2 f$ & - & - & - & - & - & - \\
\hline $2 g$ & - & - & - & - & - & - \\
\hline 3a & 12 & 16 & 19 & 10 & - & - \\
\hline $3 b$ & 13 & 17 & 13 & 12 & - & - \\
\hline $3 c$ & 17 & 14 & 17 & 17 & - & - \\
\hline $3 d$ & 15 & 13 & 17 & 15 & - & - \\
\hline $3 e$ & 15 & 12 & 16 & 14 & - & - \\
\hline $3 f$ & 12 & 11 & 15 & 11 & - & - \\
\hline $3 g$ & 23 & 21 & 22 & 24 & - & - \\
\hline $\begin{array}{c}\text { Ampicillin } \\
\text { (X3261) }\end{array}$ & 35 & 36 & 34 & 37 & 33 & 36 \\
\hline $\begin{array}{l}\text { Neomycin } \\
\text { (X3385) }\end{array}$ & 16 & 17 & 16 & 13 & 17 & 17 \\
\hline $\begin{array}{c}\text { Step- } \\
\text { tomycin } \\
\text { (X3385) }\end{array}$ & 11 & 12 & 10 & 21 & 12 & 12 \\
\hline
\end{tabular}


Table 11. Screening for antimicrobial activity of the compounds $\mathbf{2}$ and $\mathbf{3 .}$

\begin{tabular}{|c|c|c|c|c|c|c|}
\hline \multirow{2}{*}{ Compound } & \multicolumn{7}{|c|}{ Microorganisms and Inhibition Zone (mm) } \\
\cline { 2 - 8 } & K.p. & P.a. & E.c. & S.a. & B.s. & B.c. \\
\hline $2 \mathrm{a}$ & - & - & - & - & - & - \\
\hline $2 \mathrm{~b}$ & - & - & - & - & - & - \\
\hline $2 \mathrm{c}$ & - & - & - & - & - & - \\
\hline $2 \mathrm{~d}$ & - & - & - & - & - & - \\
\hline $2 \mathrm{e}$ & - & - & - & - & - & - \\
\hline $2 \mathrm{f}$ & - & - & - & - & - & - \\
\hline $2 \mathrm{~g}$ & - & - & - & - & - & - \\
\hline $3 \mathrm{a}$ & ++ & ++ & +++ & + & - & - \\
\hline $3 \mathrm{~b}$ & ++ & +++ & ++ & ++ & - & - \\
\hline $3 \mathrm{c}$ & +++ & ++ & +++ & +++ & - & - \\
\hline $3 \mathrm{~d}$ & ++ & ++ & +++ & ++ & - & - \\
\hline $3 \mathrm{e}$ & ++ & ++ & ++ & ++ & - & - \\
\hline $3 \mathrm{f}$ & ++ & ++ & ++ & ++ & - & - \\
\hline $3 \mathrm{~g}$ & +++ & +++ & +++ & +++ & - & - \\
\hline
\end{tabular}

The inhibition zone: (-): $<5.5 \mathrm{~mm}$; (+): 5.5-10 mm; (++): 11-16 mm; (+++): $\geq 17 \mathrm{~mm}$.

K.p.: Klepsiella pneumoniae (ATCC4352), P.a.: Pseudomonas aeruginosa (ATCC27853), E.c.: Escherichia coli (ATCC25922). S.a.: Staphylococcus aureus (ATCC6538), B.s.: Bacillus subtilis (ATCC11774), B.c.: Bacillus cereus (ATCC11778).

All of the Schiff Bases (2a-g) showed no effect against six bacteria. All of the Mannich Bases (3a-g) showed no effect against B. Subtilis ATCC11774 and B. cereus ATCC11778 bacteria strains. The antimicrobial activity of $\mathbf{3 a - g}$ compounds against $K$. pneumoniae, $P$. Aeruginos, E. coli, $S$. aureus is lower than Ampicillin and higher than Neomycin and Streptomycin standards and listed in Table 1o. While compounds $\mathbf{3 b}, \mathbf{3 g}$ showed high activity against P. Aeruginosa ATCC27853, compounds $3 \mathbf{a}, \mathbf{3 c}, \mathbf{3 d}$, $3 \mathbf{e}$ and $\mathbf{3}$ f showed moderate activity to this strain. On the other hand, different results were obtained from $K$. pneumoniae ATCC4352 strain. While compounds 3c, $3 \mathbf{g}$ showed high activity against K. pneumoniae ATCC4352, Staphylococcus aureus (ATCC6538) and Escherichia coli (ATCC25922) compounds $\mathbf{3 a}, \mathbf{3 b}, \mathbf{3 d}, \mathbf{3 e}$ and $\mathbf{3} \mathbf{f}$ showed moderate activity to this strain. The compound $\mathbf{3 a}, \mathbf{3 c}, \mathbf{3 d}, \mathbf{3 g}$ showed high activity against Escherichia coli (ATCC25922) but, compound 3b, 3e, 3f showed moderate activity against same bacteria. Compound $\mathbf{3 g}$ showed high activity against first four bacteria and summarized in Table 10, $\mathbf{1 1}$.

The synthesis and in-vitro antioxidant valuation of fourteen new compounds 
are explained. Antioxidant activity (the metal chelate activity) and anti-microbial activity of Mannich bases (3a-g) were higher than Schiff base compounds (2a-g). Synthesis of these new Mannich Bases can play especially a safety role in modern medicinal chemistry. These results may also ensure some lead for the improving of Mannich Bases curative aim. 


\section{REFERENCES}

1. Tramontini, M.; Angiolini, L. Mannich Bases. Chemistry and Uses. CRC Press. 1994, 289.

2. Savariz, F. C.; Formagio, A. S. N.; Barbosa, V. A.; Foglio, M. A.; Carvalho, J. E. De.; Duarte, M. C. T. Synthesis, antitumor and antimicrobial activity of novel 1-substituted phenyl-3-[3alkylamino(methyl)-2-thioxo-1,3,4-oxadiazol-5-yl] $\beta$-carboline derivatives. J. Braz Chem Soc. 2010, 21, 288-98.

3. Chen, Y.; Wang, G.; Duan, N.; Cao, T.; Wen, X.; Yin, J. Synthesis and Antitumor Activity of Fluoroquinolone C3-Isostere Derivatives: Oxadiazole Mannich Base Derivatives. Chinese J. Appl. Chem. 2012, 29, 1246-1250.

4. Bandgar, B. P.; Patil, S. A.; Korbad, B. L.; Biradar, S. C.; Nile, S. N.; Khobragade, C. N. Synthesis and biological evaluation of a novel series of 2,2-bisaminomethylated aurone analogues as anti inflammatory and antimicrobial agents. Eur. J. Med. Chem. 2010, 45, 3223-3227.

5. El-Emam, A. A.; Al-Tamimi, A-M. S.; Al-Omar, M. A.; Alrashood, K. A.; Habib, E. E. Synthesis and antimicrobial activity of novel 5-(1-adamantyl)-2-aminomethyl-4-substituted-1,2,4triazoline-3-thiones. Eur. J. Med. Chem. 2013, 68, 96-102.

6. Maddila, S.; Jonnalagadda, S. B. New Class of Triazole Derivatives and Their Antimicrobial Activity. Lett. Drug. Des. Discov. 2012, 9, 687-693.

7. Das, S.; Das, U.; Bandy, B.; Gorecki, D. K. J.; Dimmock, J.R. 2-[4-(4-Methoxyphenylcarbonyloxy)benzylidene]-6-dimethylaminomethyl cyclohexanone hydrochloride: a Mannich base which inhibits the growth of some drug-resistant strains of Mycobacterium tuberculosis. Pharmazie, 2010, 65, 849-850.

8. Sriram, D.; Yogeeswari, P.; Gopal, G. Synthesis, anti-HIV and antitubercular activities of lamivudine prodrugs. Vol. 40. Eur. J. Med. Chem. 2005, 4O, 1373-1376.

9. Ceylan, S.; Bektas, H.; Bayrak, H.; Demirbas, N.; Alpay-Karaoglu, S.; Ülker, S. Syntheses and Biological Activities of New Hybrid Molecules Containing Different Heterocyclic Moieties. Arch. Pharm. (Weinheim). 2013, 346, 743-756.

10. Liu, D.; Yu, W.; Li, J.; Pang, C.; Zhao, L. Novel 2-(E)-substituted benzylidene-6-(N-substituted aminomethyl)cyclohexanones and cyclohexanols as analgesic and anti inflammatory agents. Med. Chem. Res. Springer-Verlag. 2013, 22, 3779-3786.

11. Bandgar, B. P.; Patil, S. A.; Totre, J. V.; Korbad, B. L.; Gacche, R. N.; Hote, B. S. Synthesis and biological evaluation of nitrogen-containing benzophenone analogues as TNF- $\alpha$ and IL-6 inhibitors with antioxidant activity. Bioorg. Med. Chem. Lett. 2010, 20, 2292-2296.

12. Köksal, M.; Gökhan, N.; Küpeli, E.; Yesilada, E.; Erdoğan, H. Synthesis, analgesic and anti inflammatory properties of certain 5-/6-acyl-3-(4-substituted-1-piperazinylmethyl)-2-benzoxazolinones derivatives. Arch. Pharm. (Weinheim). 2005, 338, 117-125.

13. Nithinchandra.; Kalluraya, B.; Aamir, S.; Shabaraya, AR. Regioselective reaction: Synthesis, characterization and pharmacological activity of some new Mannich and Schiff bases containing sydnone. Eur. J. Med. Chem. 2012, 54, 597-604.

14. Manjunatha, K.; Poojary, B.; Lobo, P. L.; Fernandes, J.; Kumari, N.S. Synthesis and biological evaluation of some 1,3,4-oxadiazole derivatives. Eur. J. Med. Chem. 2010, 45, 5225-5233.

15. Ozkan-Daguyan, I.; Sahin, F.; Koksal, M. Synthesis, Characterization and Antimicrobial Activity of Novel 3,5-Disubstituted-1,3,4-oxadiazole-2-ones. Rev. Chim. Orig. Ed. 2013, 64, 534-539.

16. Frank, P. V.; Manjunatha Poojary, M.; Damodara, N.; Chikkanna, C. Synthesis and anti- 
microbial studies of some Mannich bases carrying imidazole moiety. Acta Pharm. 2013, 63, 231-239.

17. Pati, H. N.; Das, U.; Kawase, M.; Sakagami, H.; Balzarini, J.; De Clercq, E. 1-Aryl-2-dimethylaminomethyl-2-propen-1-one hydrochlorides and related adducts: A quest for selective cytotoxicity for malignant cells. Bioorg. Med. Chem. 2oo8, 16, 5747-5753.

18. Pau, A.; Murineddu, G.; Asproni, B.; Murruzzu, C.; Grella, G. E.; Pinna, G. Synthesis and cytotoxicity of novel hexahydrothienocycloheptapyridazinone derivatives. Molecules. 20o9, 14, 3494-3508.

19. Jia, W.; Zhao, Y.; Li, R.; Wu, Y.; Li, Z.; Gong, P. Synthesis and In-Vitro Anti-HepatitisB Virus Activity of 6H-[1]Benzothiopyrano[4,3-b] quinolin-10-ols. Arch. Pharm (Weinheim). 2009, 342, 507-512.

20. Jia, W.; Liu, Y.; Li, W.; Liu, Y.; Zhang, D.; Zhang, P. Synthesis and in vitro anti-hepatitis B virus activity of 6H-[1] benzothiopyrano[4,3-b]quinolin-9-ols. Bioorg. Med. Chem. 2009, 17, 4569-4574.

21. Chen, D.; Zhai, X.; Yuan, Q. H.; Luo, J.; Xie, S. C.; Gong, P. Synthesis and in vitro anti-hepatitis B virus activity of $1 \mathrm{H}$-benzimidazol-5-ol derivatives. Chin Chem Lett. 2010, 21, 1326-1329.

22. Köksal, M.; Bilge, S. S. Synthesis and Antidepressant-Like Profile of Novel 1-Aryl-3-[(4benzyl)piperidine-1-yl]propane Derivatives. Arch Pharm (Weinheim). 2007, 340, 299-303.

23. Dyubchenko, O. I.; Nikulina, V. V.; Markov, A. F.; Kandalintseva, N. V.; Prosenko, A. E.; Khoshchenko, O. M. Synthesis and hepatoprotector activity of water-soluble derivatives of aminoalkylphenols. Pharm. Chem. J. 2006, 4O, 243-247.

24. Kodhati, V.; Vanga, M. R.; Yellu, N. R. Synthesis and Anti Bacterial and Anti-ulcer Evaluation of New S-mannich Bases of 4,6-diaryl-3,4-dihydropyrimidin-2(1H)-thiones. J. Korean Chem. Soc. 2013, 57, 234-240.

25. Rajasekaran, A.; Rajamanickam, V.; Darlinquine, S. Synthesis of some new thioxoquinazolinone derivatives and a study on their anticonvulsant and antimicrobial activities. Eur. Rev. Med. Pharmacol Sci. 2013, 17, 95-104.

26. Görlitzer, K.; Kramer, C.; Meyer, H.; Walter, R. D.; Jomaa, H.; Wiesner, J. Pyrido [3,2-b] indol-4-yl-amine-Synthese und Prufung auf Wirksamkeit gegen Malaria. Pharmazie. 2oo4, $59,243-250$.

27. Görlitzer, K.; Meyer, H.; Walter, R. D.; Jomaa, H.; Wiesner, J. [1] Benzothieno[3,2-b]pyridin-4-yl-amine-Synthese und Prufung auf Wirk-samkeit gegen Malaria. Pharmazie. 2004, 59, 506-512.

28. Hamama, W. S.; Zoorob, H. H.; Gouda, M. A.; Afsah, E.M. Synthesis and antimicrobial and antioxidant activities of simple saccharin derivatives with N-basic side chains. Pharm. Chem. $J$. 2011, 45, 18-24.

29. Hussain, H. H.; Babic, G.; Durst, T.; Wright, J. S.; Flueraru, M.; Chichirau, A. et al. Development of novel antioxidants: design, synthesis, and reactivity. J. Org. Chem. 2003, 68, 7023-7032.

30. McClements, D.; Decker, E. Lipid oxidation in oil-in-water emulsions: Impact of molecular environment on chemical reactions in heterogeneous food systems. J. Food Sci. 200o, 65, 1270-1282.

31. Gürsoy-Kol, Ö.; Yuksek, H. Synthesis and In Vitro Antioxidant Evaluation of Some. E-Journal Chem. 2010, $7,123-136$. 
32. Perez, C.; Pauli, M.; Bazerque, P. An antibiotic assay by agar-well diffusion method. Acta Biol. Med. Exp. 1990, 15, 113-115.

33. Ikizler, A.; Yüksek, H. Acetylation of 4-amino-4,5-dihydro-1H-1,2,4-triazol-5-ones. Org. Prep. Proced Int. 1993, 25, 99-105.

34. Ikizler, A.; Un, R. Reactions of ester ethoxycarbonylhydrazones with some amine type compounds. Chim. Acta Turcia. 1979, 7, 269-290.

35. Oyaizu, M. Studies on products of browning reaction. Antioxidative activities of products of browning reaction prepared from glucosamine. Japanese J. Nutr Diet. 1986, 44, 307-315.

36. Blois, M. Antioxidant Determinations by the Use of a Stable Free Radical. Nature. 1958, 181, 1199-1200.

37. Dinis, T. C. P.; Madeira, V. M. C.; Almeida, L. M. Action of Phenolic Derivatives (Acetaminophen, Salicylate, and 5-Aminosalicylate) as Inhibitors of Membrane Lipid Peroxidation and as Peroxyl Radical Scavengers. Arch. Biochem Biophys. 1994, 315, 161-169.

38. Ahmad, I.; Mehmood, Z.; Mohammad, F. Screening of some Indian medicinal plants for their antimicrobial properties. J. Ethnopharmacol. 1998, 62, 183-193.

39. Meir, S.; Kanner, J.; Akiri, B. Philosophhadas S, Rk. Determination and involvement of aqueous reducing compounds in oxidative defense systems of various senescing leaves. $J$. Agric. Food Chem. 1995, 43, 1813-1819.

40. Yildirim, A.; Mavi, A.; Kara, A. A. Determination of antioxidant and antimicrobial activities of Rumex crispus L. extracts. J. Agric. Food Chem. 2001, 49, 4083-4089.

41. Baumann, J.; Wurn, G.; Bruchlausen, V. Prostaglandin synthetase inhibiting O2- Radical scavenging properties of some flavonoids and related phenolic compounds. Naunyn-Schmiedebergs Arch. Pharmacol. 1979, 308, R27.

42. Soares, J. R.; Dinis, T. C. P.; Cunha, A. P.; Almeida, L. M. Antioxidant activities of some extracts of Thymus zygis. Free Radic Res. 1997, 26, 469-478.

43. Duh, P.; Tu, Y.; Yen, G. Antioxidant activity of water extract of harn jyur (Chrysanthemum morifolium Ramat). Leb Wissen Technol. 1990, 32, 269-277.

44. Yamaguchi, F.; Ariga, T.; Yoshimura, Y.; Nakazawa, H. Antioxidative and anti-glycation activity of garcinol from Garcinia indica fruit rind. J. Agric. Food Chem. 2ooo, 48, 180-185 